\title{
GALV ou la synchronisation des espaces du militantisme breton
}

GALV, or the synchronisation of spaces of Breton activism

\section{Mathilde Sempé}

\section{(2) OpenEdition}

\section{Journals}

Édition électronique

URL : https://journals.openedition.org/lbl/463

DOI : $10.4000 / \mathrm{lbl} .463$

ISSN : 2727-9383

\section{Éditeur}

Université de Bretagne Occidentale - UBO

\section{Édition imprimée}

Date de publication : 30 décembre 2019

Pagination : 45-60

ISBN : 979-10-92331-49-3

ISSN : 1270-2412

Référence électronique

Mathilde Sempé, « GALV ou la synchronisation des espaces du militantisme breton », La Bretagne Linguistique [En ligne], 23 | 2019, mis en ligne le 01 mai 2020, consulté le 04 mai 2021. URL : http:// journals.openedition.org/lbl/463; DOI : https://doi.org/10.4000/lbl.463



La Bretagne Linguistique est mise à disposition selon les termes de la Licence Creative Commons Attribution 4.0 International. 


\title{
GALV ou la synchronisation des espaces du militantisme breton
}

MATHILDE SEMPÉ*

\begin{abstract}
«On comptait sur les jeunes: ils vinrent, mais avec leurs professeurs, avec leurs aînés, leurs camarades ouvriers et paysans. [...]. Ar Falz apportait son expérience, la JEB. ses meilleures analyses, notre Parti, sa rigueur et sa maturité politique ${ }^{1}$.»
\end{abstract}

T a pétition, lancée par les membres d'Emgleo Breiz ${ }^{2}$ en 1967, est Là l'origine de la création, le 2 mars 1969, d'un Comité d'action progressiste pour la langue Bretonne $\left(\mathrm{CALB}^{3}\right)$, réunissant à Brest les adhérent-e-s d'Ar Falz (La faucille), de la Jeunesse étudiante bretonne (JEB) et de l'Union démocratique bretonne (UDB), respectivement revue/association culturelle, association étudiante et parti politique de promotion de la langue et de la culture bretonnes situés à gauche du clivage politique. Ainsi que le précisent les militant-e-s, "tous les progressistes amis de la Bretagne et de la langue bretonne" sont appelés «à se regrouper en vue d'engager une action massive en faveur de la décolonisation culturelle du pays, car seule la France, comme l'Espagne et la Grèce, refuse toute possibilité d'existence légale aux

\footnotetext{
* Docteure en science politique, Université Paris-Nanterre (ISP, UMR 7220) mathildesempe@gmail.com.

1. Le peuple Breton (organe de presse de l'UDB), Le peuple Breton, n 68, mai 1969, cité dans $\mathrm{Ar} \mathrm{Falz}$, n 2, ebrel-mae (avril-mai) 1969, $4^{\mathrm{e}}$ de couverture, consulté dans les collections de la bibliothèque des Champs Libres à Rennes.

2. Fédération d'associations dites de "culture populaire», créée en 1955.

3. En breton: Kuzul stourmerien ar brezoneg. Le siège du CALB est fixé à Brest, il s'agit également du siège de la section de Brest de la JEB.
} 
langues parlées sur son territoire ${ }^{4}$ ». De nouveau réunis à Brest le 21 mars 1969, les responsables du collectif entendent poursuivre la lutte "pour la reconnaissance de la langue bretonne et contre la politique de colonisation et de génocide culturel menée en Bretagne ${ }^{5}$ ». Enfin, le 18 mai 1969, le premier congrès du CALB - désormais dénommé GALV (l'appel) - rassemble à Brest 500 militant-e-s ${ }^{6}$ mobilisés en faveur de l'institutionnalisation de la "culture populaire» bretonne, dont la reconnaissance étatique d'un enseignement et d'une diffusion radiophonique en langue bretonne permettrait d'en assurer la légitimation ${ }^{7}$.

Si les déterminants socioprofessionnels (les capitaux linguistiques, culturels, politiques hérités et acquis, la profession enseignante ou encore les engagements associatif et politique) ainsi que la multipositionnalité des agents sociaux qui composent ces trois organisations permettent en partie d'expliquer la rencontre d'espaces ordinairement distincts du monde social (littéraire, étudiant et politique), quels enjeux et conditions sociohistoriques permettent également de rendre compte de la formation de GALV et, en cela, de la synchronisation de l'espace du militantisme breton en 1969? Après avoir restitué la genèse des trois organisations fondatrices de GALV et des enjeux qui les structurent, nous montrerons en quoi les usages d'un répertoire d'actions collectives anticolonialiste ainsi que la conjoncture spécifique de crise politique de la fin des années 1960, contribuent à la compréhension d'un décloisonnement circonstanciel d'espaces du militantisme breton auparavant différenciés.

4. «Titre IV: Galv (l'appel), $2^{\mathrm{e}}$ partie», dans «IVLe mouvement politique Breton de janvier 1969 à mars 1972 », Cabinet du préfet, Archives d'Ille-et-Vilaine, 508W153, p. 55.

5. Ibid.

6. Ar Falz, 1969, op. cit., p. 4.

7. Interviennent au nom des trois organisations: Armand Keravel (secrétaire général d'Ar Falz), Jean-Yves Lagadec (président de la JEB), Erwan Evenou (membre de l'UDB) et Jean-Claude Léon (chargé des relations extérieures du bureau central de l'UDB) alors président de séance de GALV. 


\section{Genèse des organisations et enjeux de luttes pour un enseignement en langue bretonne}

Au préalable d'une compréhension des enjeux de luttes pour l'institutionnalisation d'un enseignement et d'une radio en langue bretonne, il convient de restituer la rapide genèse des trois groupes sociaux qui forment GALV en 1969. La revue Ar Falz, créée en 1933 par Yann Sohier ${ }^{8}$, instituteur militant pour un enseignement et une littérature en langue bretonne, est un bulletin adressé aux instituteurs laïcs. Après sa mort en 1935, Ar Falz est dirigée, jusqu’en 1939, par Yann Kerlann, également instituteur engagé en faveur de l'enseignement du breton à l'école. Destitué de ses fonctions d'enseignant en 1946, pour avoir collaboré avec l'occupant durant la seconde guerre mondiale, il est remplacé, à la Libération, par Armand Keravel ${ }^{9}$ qui devient le secrétaire général de la revue ${ }^{10}$. Ar Falz (l'association) ne reprend cependant ses activités et dépose ses statutsà la sous-préfecture de Brest qu' en mai 1947. Prétendant contribuer à "l'éducation populaire», ses membres entendent participer "à toutes les activités d'ordre culturel et d'inspiration régionaliste et laïque en Bretagne», l'association s'interdisant, par ailleurs, « de prendre position sur le terrain politique ${ }^{11}$ ». Néanmoins, à partir de 1963, et compte tenu d'une "hostilité foncière du pouvoir à satisfaire ses

8. Pour une sociogenèse d'Ar Falz et un retour sur la trajectoire biographique de Yann Sohier, voir Mathilde SEMPÉ, «La revue bretonne Ar Falz: "l'art social" en conjoncture de crise politique et littéraire (1945-1946)", COnTEXTES. Revue de sociologie de la littérature, $\mathrm{n}^{\circ} 16$, décembre 2015 [en ligne].

9. Pour un retour sur la trajectoire biographique d'Armand Keravel, également instituteur, voir Mathilde SEMPÉ, L'invention d'une identité régionale: la Bretagne et le livre (1945-2014), Thèse sous la direction d'Emmanuel Wallon, Université ParisNanterre, 2014.

10. Au terme d'enjeux de luttes pour l'appropriation de la revue, le recrutement social des membres d'Ar Falz est caractéristique de la détention d'un capital résistant et dominé par la figure de l'intellectuel engagé, parmi lesquels René-Yves Creston, Charles Ar Gall, Andreo Ar Merser et Per Jakez Helias. Ce dernier, président de l'association Ar Falz, occupe de nombreuses positions dans les champs éditorial, de la presse, de la radio et des espaces du militantisme résistant et enseignant.

11. Direction centrale des renseignements généraux, Direction générale de la Sûreté nationale, Ministère de l'Intérieur, "Les mouvements autonomistes en Bretagne», septembre 1968, Archives de la préfecture de police de Paris, Clubs régionalistes: GABR46, p. 3-4. 
revendications culturelles, notamment l'enseignement du breton ${ }^{12}$ ", les membres d'Ar Falz concourent à la formation du Comité d'action pour la Bretagne (CAB), qui regroupe le PCF, le PSU, la SFIO, la FEN, la CGT et la CFTC et les engagent notamment dans les mobilisations pour la défense des forges d'Hennebont en 1963. Lors de la création de GALV en 1969, Armand Keravel en devient le secrétaire adjoint.

La JEB est une association créée en $1952^{13}$, à l'initiative de Per Bernard ${ }^{14}$, enseignant de philosophie, dans un double contexte de promulgation, un an plus tôt, de la loi Deixonne - «relative à l'enseignement des langues et dialectes locaux ${ }^{15}$ ", qui permettait d'initier le processus d'institutionnalisation de la langue bretonne notamment au sein des universités - et d'une conjoncture économique peu favorable aux travailleurs bretons. Il s'agit alors d'informer les étudiant-e-s, par l'intermédiaire d'un bulletin de liaison (L'étudiant Breton, créé en 1954 et remplacé en 1964 par JEB-informe). En 1965, une charte (la charte d'Argol) est adoptée afin de "pallier les carences de l'enseignement en ce qui concerne la langue, l'histoire, la littérature et la civilisation bretonnes» et concourir «à l'élaboration de la culture bretonne moderne en s'inspirant notamment de la culture populaire", ses signataires refusant par ailleurs "toute adhésion collective à un quelconque parti ou mouvement politique ${ }^{16}$ ", sans pour autant exclure les adhésions individuelles, particulièrement à l'UDB.

Parti politique né de la scission du Mouvement pour l'organisation de la Bretagne (MOB), à l'initiative de Ronan Leprohon ${ }^{17}$ (aux

12. "Titre III : Ar Falz ( $2^{\mathrm{e}}$ partie branche progressiste)», dans «Le mouvement politique Breton de janvier 1969 à mars 1972 », Cabinet du préfet, Archives d'Ille-etVilaine, 508W153, p. 52.

13. La JEB devient une fédération d'associations en 1961.

14. Pierre Bernard sera le porte-parole du groupe GALV de Lorient.

15. La loi $\mathrm{n}^{\circ}$ 51-46 dite «Deixonne» du 11 janvier 1951.

16. "Titre V : La jeunesse étudiante bretonne ( $3^{\text {e }}$ partie branche gauchiste)", dans «IV Le mouvement politique Breton de janvier 1969 à mars 1972», Cabinet du préfet, Archives d'Ille-et-Vilaine, 508W153, p. 141.

17. Enseignant, Ronan Leprohon figure, en tant que représentant de l'UDB, dans le premier bureau du CALB, il sera ensuite remplacé par Erwan Evenou, également instituteur, lors du premier congrès de GALV. Voir la «Fiche de renseignements d'Yvon Evenou. Yvon dit Erwan », dans Ministère de l'Intérieur, Direction générale de la police 
relations extérieures), Jean-Yves Veillard (chargé de la presse et de la «propagande $\left.{ }^{18} »\right)$, Pierre Le Padellec et Robert Debroise, l'UDB est fondée en $1964^{19}$. "Le nouveau parti" veut rassembler, dans la mesure de ses moyens, les Bretons et les amis de la Bretagne désireux d'œuvrer pour la promotion de la personnalité bretonne sur des bases démocratiques et modernes ${ }^{20}$.» L'UDB, qui compte une trentaine de militant-e-s à sa création, apparaît, en 1968, au regard des rapports établis par les renseignements généraux, comme «déjà fortement structurée $^{21} »$. Son recrutement social est principalement opéré au sein des espaces du militantisme étudiant et des professions enseignantes (entre 130 et 150 membres environ selon les renseignements généraux) et ses membres cherchent à "promouvoir une culture populaire "engagée" pour combattre la culture actuelle de type "colonial" 22 ".

La lecture du manifeste de GALV, par Fañch Broudic - alors secrétaire général de GALV, lors du congrès fondateur du 18 mai 1969 met au jour, les revendications des militant-e-s relatives à l'enseignement de la langue bretonne ${ }^{23}$ :

nationale, Direction des renseignements généraux, «V Partis, mouvements, groupes politiques 1952-1977», Cabinet du préfet, Archives d'Ille-et-Vilaine, 508W157.

18. Direction des renseignements généraux, Direction générale de la Sûreté nationale, Ministère de l'Intérieur, "Le malaise breton », 14 mars 1964, Archives de la préfecture de police de Paris, Clubs régionalistes, GABR46, p. 45-46.

19. Voir Romain PASQUIER, "L'union démocratique bretonne ou les limites de l'expression partisane autonomiste en Bretagne», Pôle Sud, n 20, 2004, p. 113-132; T. Kernalegenn et R. PAsquier (dir.), L'Union démocratique bretonne. Un parti autonomiste dans un État unitaire, Rennes, PUR, 2014, p. 205-210.

20. «Le malaise breton», op. cit.

21. Ibid., p. 1.

22. «Titre I : l'UDB ( $2^{\text {e }}$ partie branche progressiste)», dans «IV Le mouvement politique Breton de janvier 1969 à mars 1972 », Cabinet du préfet, Archives d'Ille-etVilaine, 508W153, p. 45.

23. Faute de place, nous ne pourrons traiter des revendications liées à la création d'émissions télévisées et radiophoniques en langue bretonne. Nous préciserons toutefois, aminima, que les revendications sont les suivantes: une heure de breton par jour à la radio, la présence d'émissions bretonnes, culturelles et d'information, en français, à la radio et à la télévision; la création d'un office breton de la radio et de la télévision en concurrence de l'ORTF est également précisée. Voir Galv. Comité d'Action Progressiste pour la Langue Bretonne, Livre Blanc et Noir de la Langue Bretonne, Brest, Galv, juin 1969. 
«Les Bretons et amis de la Bretagne soussignés constatent que la langue bretonne est pratiquement exclue de l'enseignement: aucun cours n'est possible dans le premier degré, et dans le second degré les cours ne peuvent fonctionner que dans des conditions pénibles; aucune sanction sérieuse n'existe à aucun examen; l'histoire de la Bretagne et la littérature bretonne ne sont pas insérées dans les programmes ${ }^{24}$.»

Comprendre l'émergence de ces mobilisations nécessite de rappeler que le processus d'unification administrative, rendu possible par l'«apprentissage du français à l'école et par l'école ${ }^{25}$ ", tend à rendre marginale la pratique de la langue bretonne immédiatement associée à un territoire rural et économiquement archaïque ${ }^{26}$, de sorte que les langues régionales, requalifiées de patois sont immédiatement rangées dans les formes populaires de la culture ${ }^{27}$, l'incorporation d'une indignité culturelle recouvrant dès lors une indignité sociale. En effet, l'injonction linguistique n'a pu se faire que par des formes variées de consentement des agents sociaux eux-mêmes, dans l'absence de transmission des langues, parlers et dialectes locaux. Ainsi, il a fallu qu'une génération de Bretons - toutes choses étant égales par ailleurs incorpore dans leurs habitus linguistiques ${ }^{28}$ l'idée selon laquelle l'emploi de la languedominée serait immédiatement associé à des formes de dévaluation caractéristiques de la position sociale occupée, réduisant en conséquence les chances d'atteindre une place au sein des marchés scolaires et du travail ${ }^{29}$. Et la diffusion de la croyance en ce que la langue française est la langue d'enseignement, a fonctionné dès lors qu'elle a touché les catégories sociales les plus hautes, celles qui disposent des

24. «Titre IV : Galv (l'appel), $2^{\text {e }}$ partie», dans «IV Le mouvement politique Breton de janvier 1969 à mars 1972», Cabinet du préfet, Archives d'Ille-et-Vilaine, 508W153, p. 55-65.

25. Maurice AgulHon, «Le centre et la périphérie», dans P. Nora (dir.), Les lieux de mémoire. Les France, Paris, Gallimard, 1997, p. 831. Les lois Ferry de 1886 ont mis en place l'école laïque et rendu obligatoire l'instruction par l'usage de la langue française. 26. Voir Catherine BERTHO, "Linvention de la Bretagne», Actes de la recherche en sciences sociales, vol. 35, 1980, p. 45-62.

27. Pierre BOURDIEU, «La production et la reproduction de la langue légitime», Langage et pouvoir symbolique, Paris, Fayard, 2001 (1982), p. 73.

28. Pierre Bourdieu et Luc BoltansKI, "Le fétichisme de la langue», Actes de la recherche en sciences sociales, vol. $1, \mathrm{n}^{\circ} 4,1975$, p. 8.

29. Ibid., p. 9. 
moyens symboliques de domination pour l'imposer comme telle (par la concentration des ressources culturelles, sociales et économiques). C'est pourquoi, le renversement des stigmates, par la défense d'une culture explicitement labellisée comme populaire, à partir de la Libération, a dans le même temps questionné les mécanismes sociaux de dépossession de la parole sur soi. C'est ce que met en évidence JeanClaude Léon, intervenant au nom de GALV, lors du congrès du 18 mai 1969 lorsqu'il précise que "la bourgeoisie avait besoin, a toujours besoin, aura toujours besoin d'un peuple [...] dépossédé de pouvoir [...] dépossédé de sa langue ${ }^{30}$ ", et que confirment les propos publiés dans le Livre Blanc et Noir de la Langue Bretonnecette même année: "La bourgeoisie française au pouvoir, en méprisant et en opprimant la langue bretonne, tout en exploitant économiquement le peuple breton, ne tend en réalité qu'à perpétuer sa domination de classe dirigeante sur les classes défavorisées de Bretagne ${ }^{31}$.» C'est donc au terme d'un long processus de légitimation de la langue et de la culture bretonnes - initié à la fin du XIXe siècle et réifié dans les années $1950^{32}$, par des agents des espaces du militantisme enseignant ${ }^{33}$, littéraire, éditorial et politique bretons - que les prises de position politiques des différentes organisations culturelles et politiques bretonnes, situées à gauche du clivage politique ${ }^{34}$, tendent à correspondre à une conjoncture nationale

30. Jean-Claude Léon, intervention au nom de GALV, lors du congrès de GALV du 18 mai 1969, cité dans Ar Falz, 1969, op. cit., p. 11.

31. Galv. Comité d'Action Progressiste pour la Langue Bretonne, Livre Blanc et Noir de la Langue Bretonne, op. cit., p. 21.

32. Voir M. SEMPÉ, L'invention d'une identité régionale: la Bretagne et le livre (19452014), op. cit.

33. Faute de place, nous ne pourrons établir ici une prosopographie des militante-s, néanmoins, il convient de préciser que la profession enseignante est une variable sociologique déterminante de l'engagement.

34. Selon les taxinomies élaborées par les préfets d'Ille-et-Vilaine et de région, les trois organisations appartiendraient à «la branche gauchiste et progressiste du Mouvement Breton", dans «IV Le mouvement politique Breton de janvier 1969 à mars 1972», Cabinet du préfet, Archives d'Ille-et-Vilaine, 508W153, p. 4. 
de remise en cause de l'ordre symbolique établi et notamment de l'ordre scolaire ${ }^{35}$.

À cet égard, si les images véhiculées des Bretons à la fin du XIX siècle dans les manuels scolaires favorisent tendanciellement, selon AnneMarie Thiesse, la formation et l'intériorisation d'une indignité sociale et culturelle ${ }^{36}$, il apparaît cependant que l'enseignement de l'histoire locale, en fonction de celui de l'histoire nationale diffère selon la pluralité des cas d'espèce et selon les acteurs et les rapports de force en présence (inspecteurs d'académie, enseignant-e-s, structures familiales, etc.). Il en est de même à propos des modalités de standardisation de l'emploi de la langue française, là où précisément elle n'était pas normalisée. L'exemple des écoles normales d'instituteurs (ENI), étudié dans l'ouvrage de JeanFrançois Chanet, montre à quel point les aménagements sont tributaires des réalités de terrain. Professeur-e-s et directeurs d'établissements scolaires prenaient ainsi en considération, selon les configurations, les inégalités d'accès à la «langue commune ${ }^{37}$ ». Cela ne contribue pas pour autant à la légitimation institutionnelle de la langue bretonne. Aussi est-il précisé, par les membres de GALV, dans le Livre Blanc et Noir de la Langue Bretonne, la nécessité de l'institutionnalisation de trois heures hebdomadaires facultatives de breton en Basse-Bretagne, la parité du breton dans l'enseignement et les examens vis-à-vis des autres langues vivantes et l'intégration, au sein des programmes scolaires, de l'étude

35. Voir Bernard PUDAL, "Ordre symbolique et système scolaire dans les années 1960 », dans D. Damamme, B. Gobille, F. Matonti et B. Pudal (dir.), Mai-Juin 68, Paris, Éditions de l'Atelier/Éditions ouvrières, 2008, p. 62-74.

36. Anne-Marie THIESSE, Ils apprenaient la France. L'exaltation des régions dans le discours patriotique, Paris, Éditions de la Maison des sciences de l'homme, Mission du Patrimoine ethnologique, 1997, p. 37-38.

37. Jean-François CHANET, L'École républicaine et les petites patries, Mayenne, Aubier, 1996, p. 120. Ce sont les ENI qui deviennent, à partir des années 1920, un des terreaux des nouvelles façons de penser le local et notamment par la formation des folkloristes, la mise en place de concours régionaux et nationaux, l'étude de monographies locales, des "arts et traditions populaires", dans une optique, à partir des années 1930, de conservation du patrimoine, fournissant dès lors, les ressources nécessaires à la remise en cause des schèmes propres à l'institution scolaire, celle-là même qui a en partie permis de les acquérir. Selon Anne-Marie Thiesse, les instituteurs participent à ces constructions et circulations scientifiques. Voir A.-M. THIESSE, Ils apprenaient la France, op. cit., p. 106. 
de la civilisation de la Bretagne en histoire, économie, géographie et littérature ${ }^{38}$.

Si en 1971, lors du second congrès de GALV ${ }^{39}$, est mentionnée la participation de 6000 "jeunes" à des cours de breton, il est par ailleurs précisé que les enseignant-e-s ne sont ni formés ni payés pour les dispenser; que bien qu'enseigné dans le secondaire, le breton n'en demeure pas moins absent des examens du BEPC et BEP, qui, selon les militant-e-s, sont les «seuls diplômes auxquels peuvent prétendre, bien souvent hélas! les jeunes des milieux bretonnants ${ }^{40} »$. Ainsi, pour Erwan Evenou:

"De tels cours, dans le jargon pédagogique ont un nom: on les appelle des cours clandestins! Déjà dans chacun des départements bretons, des centaines et des centaines d'étudiants et d'adultes participent à ces cours clandestins. Bien plus, il existe trois instituts d'enseignement du breton par correspondance, qui totalisent près de 1000 élèves clandestins. Ainsi, nous sommes désormais des milliers de clandestins en Bretagne ${ }^{41}$ !»

Le 22 juin 1971, une circulaire rectorale précise que le breton sera enseigné dans le secondaire, à raison de trois heures par semaine dès lors qu'un minimum de dix élèves aura été atteint ou encore que l'enseignement du breton dans le $1^{\text {er }}$ cycle et le $2^{\text {nd }}$ sera rémunéré selon certaines conditions ${ }^{42}$. Malgré la reconnaissance institutionnelle d'un certain nombre de revendications, les membres de GALV, réunis à Carhaix le 26 septembre 1971, poursuivent les mobilisations pour la rémunération des enseignant-e-s dans les autres formations ${ }^{43}$.

Les revendications pour un enseignement et une diffusion (télévisée et radiophonique) du breton ne peuvent être cependant complètement saisies qu'au regard de la considération d'une configuration inter-

38. Galv. Comité d'Action Progressiste pour la Langue Bretonne, Livre Blanc et Noir de la Langue Bretonne, op. cit., p. 37.

39. Fañch Broudic, ancien secrétaire général est remplacé par Gabriel Mer (JEB); Armand Keravel est secrétaire adjoint, dans "Titre IV : Galv (l'appel), $2^{\mathrm{e}}$ partie», op. cit., p. 55-65.

40. Ibid.

41. Erwan Evenou, au nom de l'UDB, lors du congrès de GALV du 18 mai 1969, cité dans Ar Falz, 1969, op. cit., p. 10.

42. «Titre IV : Galv (l'appel), $2^{\mathrm{e}}$ partie», op. cit., p. 63.

43. Telles que les lycées agricoles, CEG, CES et CET, dans ibid., p. 64. 
nationale singulière de prises de position anticolonialistes ainsi que d'une conjoncture nationale de crise politique, celle de la fin des années 1960.

Répertoire d'actions collectives anticolonialiste et conjoncture de crise politique: les conditions de possibilité de la synchronisation de l'espace des mouvements sociaux breton

La présence d'un vocabulaire de référence aux luttes anticolonialistes, dans les productions matérielles militantes de GALV, tant au sein du Livre Blanc et Noir de la Langue Bretonne qu'au cours des interventions lors des congrès organisés par ses membres, procède d'un contexte international pour lequel le processus de décolonisation joue un rôle dans la transformation des représentations dominantes jusqu'alors véhiculées. Ainsi, les mobilisations en faveur dela décolonisation composent des structures d'opportunité politique pour la mise au jour des revendications culturelles résultant des minorités, tant ils impulsent au retournement des stigmates et tant ils sont des marqueurs des trajectoires militantes (dans les années 1950-1960) par la co-construction et l'identification sociale à la figure de l'opprimé. L'anticolonialisme procède d'une circulation circulaire de la critique sociale des modèles colonisateurs, mais surtout d'un décloisonnement des champs sociaux, grâce à la concomitance de dispositifs sociaux d'objectivation des modes de penser et des usages sociaux qui relèvent de l'objectivé. Aux États-Unis, la guerre du Vietnam fait l'objet de contestations (à partir de 1965) qui prennent la forme de manifestations anticolonialistes (et anti-impérialistes). En France, le conflit en Indochine (qui prend fin par les accords de Genève en 1954), la transformation de l'Afrique occidentale et de l'Afrique équatoriale françaises en États indépendants (1958-1960), non moins que la guerre d'Algérie (1954-1962), donnent lieu à des changements de perception des rapports de domination, voire à des mobilisations collectives qui agissent, selon Boris Gobille, comme un «levier de politisation ${ }^{44}$ " des agents sociaux mobilisés ${ }^{45}$. En outre,

\section{Boris Gobille, Mai 68, Paris, La Découverte, 2008, p. 15.}

45. Il est d'ailleurs fait référence, dans le Livre et Blanc et Noir de la Langue Bretonne, à la déclaration générale du congrès culturel de La Havane en 1968, de même que sont évoqués les exemples de la Grèce, de l'Espagne, du Vietnam, ou encore du Pays de Galles. 
l'injonction à prendre position pour ou contre la guerre d'Algérie, polarise les espaces du politique, participe à la politisation des étudiante-s et notamment à la naissance de groupes tels que le Parti socialiste unifié (PSU) en 1960 ou encore l'UDB en $1964^{46}$. Dans cette mesure, la configuration propre au renversement des formes dominantes de la culture française, qui passent indubitablement par la langue française, constitue des structures d'opportunité politique pour la valorisation des langues minoritaires ${ }^{47}$. La Bretagne est ainsi associée - dans les discours des militant-e-s - à un "pays colonisé» et les Bretons issus des classes populaires apparaissent comme les victimes d'un "génocide culturel ${ }^{48}$ ». Aussi les membres de GALV décident-ils d'engager un combat "progressiste» pour la "décolonisation culturelle de la Bretagne». Armand Keravel entend "arracher pour la Bretagne le régime accordé aux ethnies minoritaires dans tous les pays démocratiques", Erwan Evenou appelle à mettre un terme à l'«impérialisme culturel français» et à la "politique de colonisation de la Bretagne par la bourgeoisie française», "la colonisation culturelle» n'étant, selon Jean-Yves Lagadec «que le corollaire de l'exploitation économique ${ }^{49}$ ».

«Il est intéressant de signaler par ailleurs que la France gaulliste poursuit sa politique de destruction culturelle, non seulement en Bretagne, mais aussi dans ses dernières colonies d'outre-mer. Ainsi, à la Réunion, il est rigoureusement interdit de parler le créole en classe. [...] Si l'instituteur créole lui-même parle mal le français, il aura intérêt à parler le moins possible. Car l'inspecteur veille et sanctionne ${ }^{50}$.»

46. En l'espèce, des divisions internes au MOB, relativement à la posture en défense ou non du colonisé en 1962, en viennent à ce que les événements de la guerre d'Algérie cristallisent ces tensions et comptent comme impulseurs de la construction de l'UDB. Voir Ronan LEPROHON, "L'essor d'un parti. L'UDB dans les années 1960-1970", dans T. Kernalegenn et R. Pasquier (dir.), L'Union démocratique bretonne..., op. cit., p. 206.

47. Voir Vincent Dubois, "Comment la langue devient une affaire d'État. La défense de la langue française au milieu des années 1960 », dans J. Lagroye (dir.), La politisation, Paris, Belin, 2003, p. 464.

48. «Titre IV : Galv (l'appel), 2e partie», op. cit., p. 57.

49. Ibid.

50. Galv. Comité d'Action Progressiste pour la Langue Bretonne, Livre Blanc et Noir de la Langue Bretonne, op. cit., p. 24. 
Si le processus de décolonisation peut être posé en déclencheur de démarches en déconstruction de la domination de l'État français, l'emprise de ces problématiques dans le champ politique national est doublée d'une conjoncture de légitimation de l'«illégitime» et de délégitimation du légitime, qui font que le Portrait du colonisés1 trouve un écho dans celui de "l'ouvrier», du «jeune», de «la femme», de "l'élève», du "salarié» ${ }^{52}$. En ce sens, les "crises coloniales» sont, à l'avenant, des moments de socialisation militante et intellectuelle pour toute une génération de militant-e-s anticoloniaux. Moments au cours desquels on acquiert des savoirs et savoir-faire spécifiques, un vocabulaire, des modes d'action inhérents à ce que Romain Bertrand appelle le «répertoire anticolonialiste ${ }^{53}$ ", déclinable dans d'autres sphères du militantisme.

Puisque la dynamique de mobilisation a pu générer des engagements anticolonialistes différenciés, l'hypothèse peut être émise que la confrontation, durant la socialisation primaire ou secondaire, aux instances colonisatrices, a pu fonctionner comme des composants plus ou moins bien incorporés dans les schèmes de perception individuels. Produits des institutions elles-mêmes, ces habitus peuvent entrer en conflit avec ceux acquis au sein d'autres agences de socialisation (la famille, la profession, l'Armée, l'École, etc.). Et ce sont ces tensions, entre les différentes dispositions issues de la socialisation, qui peuvent parfois occasionner le passage à l'acte militant ${ }^{54}$. Par exemple, les voyages de certains militant-e-s bretons, au sein des colonies françaises, $\mathrm{du}$ fait de la profession du père dans la marine nationale, ont pu véhiculer des représentations propres à l'administration coloniale

51. Nous faisons référence ici à l'ouvrage d'Albert MEMMI, Portrait du colonisé, Paris, Buchet-Chastel, 1957. Voir aussi du même auteur, qui paraît, précisément en 1968 : L’homme dominé, Paris, Payot, 1968.

52. Voir Dominique MEMMI, "Mai 68 ou la crise de domination rapprochée», dans D. Damamme, B. Gobille, F. Matonti et B. Pudal (dir.), op. cit, p. 35-46.

53. Romain BERTRAND, «Mai 68 et l'anticolonialisme», dans ibid., p. 95.

54. Olivier FILLIEULE, «Propositions pour une analyse processuelle de l'engagement individuel ", Revue française de science politique, vol. 51, n 1, 2001, p. 207. Dans son article l'auteur s'appuie sur les travaux de Philippe GOTTRAUX, Socialisme ou Barbarie. Un engagement politique et intellectuel dans la France de l'après-guerre, Lausanne, Payot, 1997. 
a priori tournée vers une universalisation de la culture française, aux dépens de formes concurrentes alors présentées comme mineures, et la confrontation à des modes de pensée (la culture) antagonistes a pu de surcroît s'effectuer lors de la socialisation secondaire, en particulier au moment du service militaire ou de la coopération. Cette expérience peut entraîner des conflits dans les formes de socialisation, voire des ruptures biographiques. Ainsi, la création d'une figure anticolonialiste et la circulation de théories de la domination coloniale, y compris dans les territoires d'outre-mer, n'ont pas été sans lien avec les opérations individuelles de "désobjectivation ${ }^{55}$ des rapports au monde social auparavant vécus sur le mode de l'évidence ${ }^{56}$.

À cet égard, les pratiques militantes des membres de GALV empruntent au répertoire d'actions collectives des mouvements pour les droits civiques en France. Par exemple, la marche «silencieuse et nonviolente ${ }^{57}$ » de Plouay à Lorient, du 30 mai $1971^{58}$, tend à s'inscrire dans le répertoire d'actions collectives de ce type de mouvements sociaux ${ }^{59}$. En effet, en plus de la production de pétitions, d'un manifeste, de la publication d'un bulletin mensuel d'information, de l'organisation de réunions d'information publiques ou restreintes, de la multiplication de groupes locaux, de la diffusion du Livre Blanc et Noir de la Langue Bretonne, de la création d'un macaron pour les voitures, de l'édition

55. Le terme est emprunté à Michel DOBRY, Sociologie des crises politiques, Paris, Presses de Sciences Po, 2009 (1986).

56. Un travail prosopographique ultérieur permettrait de mettre en évidence des éléments structurants des trajectoires biographiques militantes. Nous pouvons, à tout le moins, évoquer quelques variables sociologiques déterminantes de la socialisation militante du groupe social étudiételles que le capital résistant acquis ou hérité, les parents bretonnants, la socialisation politique de gauche au sein de l'institution familiale, la confrontation aux instances colonisatrices durant la coopération ou le service militaire à l'étranger et la connaissance de mouvements sociaux locaux de défense de langue et culture considérées alors comme mineures.

57. «Titre IV : Galv (l'appel), 2e partie», op. cit., p. 62.

58. Environ 700 militant-e-s seront présents à cette marche. Chiffres communiqués par le Secrétariat général d'Ar Falz, dans Ar Falz, n 2, meurz-mae (mars-mai) 1971, $4^{\mathrm{e}}$ de couverture du numéro, consulté dans les Collections de la bibliothèque des Champs Libres.

59. Voir Abdellali HAJJAT, La marche pour l'égalité et contre le racisme, Paris, Éditions Amsterdam, 2013. 
de tracts et d'affiches bilingues ou encore de l'ouverture de cours de breton dans les locaux associatifs ou municipaux ${ }^{60}$, des rassemblements et marches sont organisés par les membres de GALV ${ }^{61}$.

C'est cette conjoncture internationale anticolonialiste autant que la conjoncture nationale de crise politique de la fin des années 1960, qui permettent de comprendre les conditions de possibilité de la synchronisation des espaces du militantisme breton en 1969 au moment de la création de GALV.

En effet, l'émergence de formes critiques du "père», de «l'Église», du «maitre», du "patron", en somme, des figures dominantes des instances de socialisation - qui touche plus significativement l'ensemble des sphères du monde social à partir des années 1950 - prend sa source dans une convergence de mutations sociales et économiques occasionnant une "rupture du temps cyclique ${ }^{62}$ " en 1968, soit une modification des modes ordinaires de lecture et de classification inhérents à l'ordre social. Ainsi le progressif effacement des logiques propres à chaque secteur et la transformation des perceptions afférentes, qui ont achevé de rendre possible la rencontre des différents champs constitutifs du monde social, ne sont compréhensibles qu'en considérant cette collision dans un temps long de suspension de l'illusio par les classes parlées. "Mai-Juin 1968 " est de ces "moments critiques ${ }^{63}$ " typiques de dynamiques de mobilisations au cours desquelles un ensemble d'agents sociaux, issus de sphères hétérogènes de l'espace social, sont employés à déconstruire ce qui va de soi et la participation aux événements de 1968 des militante-s bretons est un moment d'intense politisation et de structuration des trois organisations. Pour autant, les stratégies de descriptions et de définitions des mouvements sociaux bretons, développées par les

60. Ar Falz, 1969, op. cit., $4^{\mathrm{e}}$ de couverture du numéro.

61. Évoqué par Erwan Evenou, au nom de l'UDB, lors du congrès de GALV du 18 mai 1969, cité dans ibid., p. 9. Voir aussi l'intervention de Fañch Broudic, dans ibid., p. 5. Voir aussi Jean-Claude Léon à propos du changement de répertoire d'actions collectives, dans «Titre IV : Galv (l'appel), $2^{\mathrm{e}}$ partie», op. cit., p. 58.

62. Pierre BOURDIEU, "Genèse et structure du champ religieux", Revue française de sociologie, vol. 12, $\mathrm{n}^{\circ} 3,1971$, p. 333.

63. Pierre Bourdieu, Homo academicus, Paris, Éditions de Minuit, 1992 (1984), p. 207-250. 
renseignements généraux, dans les années précédant les événements, ont tendance à les dépolitiser :

«Nés de causes hétérogènes et en des temps différents, les malaises qui ont touché les paysans, les ouvriers, les pêcheurs... ont pris en Bretagne un caractère tout à fait particulier: ils ont été rattachés à une cause générale mais unique (le sous-développement de l'Ouest) et ont conduit à une solidarité inhabituelle entre les différents secteurs sociaux. C'est à partir de cette donnée que s'explique la série de manifestations envisagées pour le mois prochain, le même jour et à la même heure, dans plusieurs villes de Bretagne. Les étudiants ont tenu à témoigner leur solidarité aux paysans et aux ouvriers. Si malaise il y a chez eux, il n’est pas spécifique de la Bretagne. Leur action n'est qu'une forme de protestation antigouvernementale. Elle ne sera pas étudiée ${ }^{64}$."

Les espaces du militantisme breton traditionnellement disposés aux revendications d'institutionnalisation de la culture et de la langue bretonnes sont, au cours de cette dynamique de «mobilisations multisectorielles ${ }^{65}$ ", au point de rencontre avec d'autres secteurs de l'espace des mouvements sociaux bretons. Les membres d'Ar Falz se sont déjà mobilisés, de 1963 à 1968, aux côtés des ouvriers des forges d'Hennebont ${ }^{66}$, contre la fermeture de l'industrie sidérurgique envisagée par l'État, se sont opposés aux licenciements dans les chantiers navals de Saint-Nazaire, entre 1963 et $1964^{67}$ et sont à l'origine de la formation du $\mathrm{CAB}^{68}$, regroupant des militant-e-s des partis de gauche en Bretagne. Par ailleurs, les étudiant-e-s des JEB sont alliés à l'UNEF, durant les mobilisations de 1968, pour la création d'un Diplôme

64. "Le malaise breton", op. cit., [n. 18], p. 25. Bien que le rapport des RG date de 1964, il apparaît que la dynamique de désectorisation des champs sociaux n'est pas strictement limitée aux événements de Mai 68. Voir P. BOURdieU, Homo academicus, op. cit., p. 210-211.

65. Michel DOBRY, op. cit.

66. Voir «Le malaise breton», op. cit., [n. 18], p. 27. Les forges d'Hennebont sont fermées en 1968.

67. Ibid., p. 28.

68. Selon la direction des RG, "cantonné jusqu'alors au seul plan culturel, "Ar Falz", à partir de 1963, amorce une ouverture politique», dans Direction centrale des renseignements généraux, Direction générale de la Sûreté nationale, Ministère de l'Intérieur, "Les mouvements autonomistes en Bretagne», op. cit., p. 4. 
d'études universitaires générales (Deug) de breton 69. De même que l'UDB s'alliera au PS et au PSU en 1977 pour former le Front culturel progressiste breton. Toutefois, c'est en 1969 que des militant-e-s des espaces littéraire (Ar Falz), étudiant (JEB) et politique (UDB) bretons contribuent à un décloisonnement spécifique de la conjoncture de la fin des années 1960, entre les champs de productions des biens politiques et culturels, comme le précise Armand Keravel, lors du congrès du 18 mai 1969: "en ce qui vous concerne, aucun doute: le combat pour la langue et la culture bretonnes est au centre du combat pour la Bretagne et il doit conduire à la victoire dans tous les autres secteurs de l'action bretonne, - secteur social et secteur politique $^{70}$ »; ainsi que le restitue également Fańch Broudic:

"C’est vrai que dans la continuité de mai 68, nous avons constitué avec deux autres associations et parti politique, en l'occurrence le mouvement Ar Falz, la JEB dont j'étais et l'UDB, un truc qui s'est appelé Comité d'Action Progressiste pour la Langue Bretonne, qui ensuite a changé de nom pour prendre une dénomination Bretonne qui s'appelle GALV et dont j'étais le secrétaire pendant deux ou trois ans. Nous avons organisé des marches, des assemblées générales, des meetings et nous avons publié un Livre Blanc et Noir de la Langue Bretonne ${ }^{71}$.»

Sans omettre les enjeux de luttes internes à l'espace du militantisme breton, ces configurations d'intense politisation que composent les contextes international de mobilisations anticolonialistes et national de crise politique concourent pour autant, de 1969 à 1971, à la synchronisation d'espaces sociaux dont les logiques militantes habituellement distinctes s'effacent un temps au profit d'une lutte collective pour la légitimation de la langue et de la culture bretonnes. Et en favorisant l'importation de modes d'actions du champ de production des biens politiques dans le champ de production des biens culturels, ce décloisonnement conjoncturel assure dans le même temps l'acquisition d'un capital militant reconvertible dans d'autres configurations, telles que le moment de négociations, avec et contre les agents de l'État, d'une Charte culturelle de Bretagne à la fin des années 1970.

69. Entretien avec Fañch Broudic, militant de la JEB, le 28 mars 2013, au Centre de Recherche Bretonne et Celtique.

70. Ar Falz, 1969, op. cit., p. 6.

71. Entretien avec Fañch Broudic le 28 mars 2013. 